\title{
SHOULDER
}

\section{Incidence of deep vein thrombosis following shoulder replacement surgery: a prospective study}

\author{
Koch $\mathrm{O}^{1}$, Du Plessis $\mathrm{A}^{2}$, Olorunju $\mathrm{S}^{3}$, McLoughlin $\mathrm{H}^{4}$, Le Roux TLB \\ FC Orth SA; Department of Orthopaedic Surgery, 1 Military Hospital, Pretoria \\ MBChB; Pretoria East Hospital \\ PhD; Biostatistics Unit, South African Medical Research Council \\ D Litt et Phil; Pretoria East Hospital \\ FCS Orth SA; Department of Orthopaedic Surgery, 1 Military Hospital, Pretoria
}

Presented in part at the COMOC in Cape Town, April 2016

Corresponding author: $\mathrm{Dr} \mathrm{O}$ Koch, Department of Orthopaedic Surgery, 1 Military Hospital, Hospital Road, Pretoria, 0187, South Africa; Email: okoch@mweb.co.za

\begin{abstract}
Background: The incidence of deep vein thrombosis (DVT) after shoulder replacement surgery is not well documented. Evidence that exists on thromboprophylaxis for upper limb surgery is based on level III and level IV studies. The hypothesis for the current study was that the incidence of DVT following shoulder replacement would be less than the published prevalence in hip and knee arthroplasties.
\end{abstract}

Methods: All participants who received shoulder arthroplasty surgery at the institution from 1 July 2013 to 30 June 2015 and who met the inclusion criteria were eligible for inclusion in the study. A duplex Doppler study was done on the affected limb of all participants on average ten days after the surgery. A study of all four limbs was done in selected participants.

Results: Fifty-seven participants (28 males and 29 females) with 30 reverse shoulder replacements, 22 hemiarthroplasties, and five resurfacing shoulder replacements were included. The incidence of DVT was $12.3 \%(7 / 57)$. Two axillary vein and three brachial vein DVTs account for the upper limb DVTs. Two DVTs were reported in the lower limb.

Conclusion: The study demonstrates that the incidence of DVT after shoulder replacement surgery was higher than anticipated and is similar to the DVT rates in lower limb arthroplasty.

Level of evidence: Level 4

Key words: shoulder replacement surgery, deep vein thrombosis, pulmonary embolism, thromboprophylaxis, venous thromboembolism

Citation: Koch O, Du Plessis A, Olorunju S, McLoughlin H, Le Roux TLB. Incidence of deep vein thrombosis following shoulder replacement surgery: a prospective study. SA Orthop J 2017;16(4):33-39. http://dx.doi.org/10.17159/2309-8309/2017/v16n4a5

Editor: Prof Anton Schepers, University of the Witwatersrand

Received: November 2016 Accepted: March 2017 Published: November 2017

Copyright: @ 2017 Koch O, et al. This is an open-access article distributed under the terms of the Creative Commons Attribution Licence, which permits unrestricted use, distribution and reproduction in any medium, provided the original author and source are credited.

Funding: Drs Koch, Du Plessis, Olorunju, McLoughlin and Le Roux did not receive any financial payments or other benefits from any commercial entity related to the subject of the article.

Conflict of interest: Drs O Koch and A du Plessis received a research grant from the South African Orthopaedic Association, sponsored by Smith \& Nephew. The grant was used in data collection. Drs S Olorunju, H McLoughlin and T le Roux declare that they have no conflict of interest. 


\section{Introduction}

The incidence of deep vein thrombosis (DVT) after shoulder replacement surgery is not well documented. Although this is a rare entity, major thrombotic complications following upper extremity DVTs, including symptomatic and fatal pulmonary embolism, have been documented. ${ }^{1-8}$

Shoulder replacement surgery requires the joint to be dislocated to allow for bone cuts, canal reaming, implant positioning and glenoid evaluation and/or preparation. The affected limb is often positioned in extreme external rotation for a prolonged period. Traction is applied to the flexed elbow. ${ }^{9}$ The hypothesis is that the traction and tortuous veins might be a precursor to the development of DVT. The axillary vein might also sustain intimal damage with the repeated internal and external rotation applied to the humerus during the procedure. ${ }^{10}$ The procedure is performed with the patient positioned seated in the so-called beach-chair position for the duration of the surgery. ${ }^{11}$ This position causes the hips to be flexed, which can potentially increase the risk of iliac vein DVTs. It also causes pooling of the blood in the lower limbs in the absence of the calf pump mechanism.

Pharmaceutical prophylaxis for venous thromboembolism (VTE) is considered the standard of care for lower limb arthroplasty. Guidelines are mostly described in lower limb arthroplasty and trauma. ${ }^{12}$ Although there is substantial proof describing optimal VTE prevention and treatment, a gap still exists in the optimal application. ${ }^{13}$ Evidence that exists on thromboprophylaxis for upper limb surgery is based on level III and level IV studies. ${ }^{10}$ Therefore, the best practice for upper limb thromboprophylaxis in unclear. From an orthopaedic surgeon's perspective, it is a delicate balance between bleeding and clotting. ${ }^{14}$ Wound-related problems and subsequent periprosthetic infection with excessive thromboprophylaxis are worrisome. ${ }^{15}$ A recent epidemiologic study by Day et al. ${ }^{6}$ concluded that the bleeding risk combined with the fewer VTE events with existing lower rates of pharmaceutical thromboprophylaxis do not justify the routine use of anticoagulation therapy in shoulder replacement surgery.

The treating surgeon can either decide to prescribe thromboprophylaxis routinely, as is advocated for lower limb arthroplasties, or individualise treatment according to the patient's risk.

This prospective study was performed to evaluate the incidence of DVT following shoulder replacement surgery and to assess whether it is possible to predict who will need prophylaxis. Venous thrombosis of the upper extremity is defined as a thrombus in the subclavian, axillary or brachial vein.

The hypothesis for the study was that the incidence of DVT following shoulder replacement surgery would be less than the published prevalence in hip and knee arthroplasties and that the patients needing routine anticoagulation can be preselected and predicted. ${ }^{16-17}$

A duplex Doppler study was done on all participants to evaluate the presence or absence of a DVT. The benign nature of the ultrasound and the relatively inexpensive cost in the clinical practice made this investigation the modality of choice. ${ }^{18}$

\section{Aims of the study}

A cross-section analytic study was performed 1) to document the incidence of DVTs in the ipsilateral limb after 57 shoulder replacements at our centre performed by two surgeons, and 2) to document DVTs in general for the study population.

The study was approved by the ethics committee of the centre in which the surgeons work. Informed written consent was obtained from each participant before study enrolment. A unique identification number was assigned to each participant to maintain participant confidentiality.

\section{Methodology}

All participants who received shoulder arthroplasty surgery (resurfacing/hemi- or reverse shoulder replacement) at the institution from 1 July 2013 to 30 June 2015 and who were able to make use of their own transport to have the duplex Doppler done, were eligible for inclusion in the study. The institution treats a large patient population which includes a majority of orthopaedic-related tumours as well as implant-related sepsis. The surgery was performed by two surgeons (OK and TLB). Patients receiving routine pre-operative anticoagulation, such as heparin or warfarin; patients with active thromboembolic disease; and patients who declined to participate in the study were excluded. All patients who were eligible were enrolled.

The surgery was done in the beach-chair position with the arm draped free in all cases. A limb positioner was not used in any of the cases done. The participants had an interscalene block before being positioned. All were treated in an arm immobiliser for six weeks post-operatively. All participants were advised to do range of motion exercises of the elbow and wrist from day one. Pendulum exercises and passive assisted external rotation were advocated for the resurfacing and hemi shoulder replacement participants. A physiotherapist helped all participants with an aggressive ambulatory programme starting on the day of surgery.

None of the patients had a central venous catheter.

A Modified Caprini Risk Assessment form ${ }^{19}$ was completed on admission by OK for all patients who agreed to participate in the study. Clinical judgement was used in the decision on whether to prescribe thromboprophylaxis. Some participants in the study population mobilised to a wheelchair or were otherwise not mobilising adequately. In these cases, thromboprophylaxis was ordered. The decision was also based on the amount of drainage recorded in the Porto-Vac after 24 h. If excessive (>200 ml), no thromboprophylaxis was prescribed. Social habits like smoking were documented. The Body Mass Index (BMI) was calculated for every participant. Participants' age and site of the previous DVT, if applicable, were individually documented.

Factors affecting surgery-related variables (operative time; surgical approach) were recorded for every participant. Documented patient-related factors were the duration of hospital stay and time to independent mobilisation defined as being able to mobilise unassisted for a distance of 50 metres. The type of replacement was recorded as this influenced the duration of surgery as well as the surgical approach. Any second surgical procedure at the time of replacement was also documented, i.e. resection of a tumour together with the replacement.

The type of anticoagulation, as well as the dosage and duration of treatment, was also recorded. No routine anticoagulation medication was used in this series. Participants who used Ecotrin ${ }^{\circledR}$ $81 \mathrm{mg}$ daily for cardiovascular or other reasons as part of their chronic medication continued with their routine medication before and after surgery. The low dose of Ecotrin ${ }^{\circledR}$ was retrospectively viewed as being an inadequate dosage. No mechanical prophylaxis was used.

Most of the participants were operated early on the list so that the physiotherapist could mobilise them actively on the same day. Their hydration status was also kept at optimum levels as far as possible via an intravenous line, and the patients were encouraged to drink adequate amounts of oral fluids. Participants also had to follow a bed programme prescribed by the physiotherapist.

A Doppler and colour flow venous duplex Doppler study was done on the affected limb of all participants within ten days of the surgery. The duplex Doppler was carried out in B-mode with compression. Compression ultrasound was performed by applying pressure to the overlying tissues to compress the visualised vein in the transverse plane. Non-compressibility of a venous segment and absence of flow defined the presence of a complete DVT. 
Partial compressibility and partial flow represent the presence of a partial DVT. The absence of flow, particularly in a vein which cannot be compressed, also suggested a DVT.

The surgeons evaluated all participants on the day of the sonographic evaluation, and clinical suspicion of a lower limb DVT was documented for further investigation. A study of all four limbs was done in selected participants. All participants were followed up for a minimum period of six weeks following surgery and any DVTrelated admissions or complications were recorded. All duplex Doppler studies were performed and interpreted by a single boardcertified musculoskeletal sonologist specialising in ultrasonography. The presence, location, timing and severity of the DVT were documented.

The computer system at the institution was reviewed for any DVTrelated admissions of all participants, should these participants have consulted at their respective units. There were no DVT-related admissions other than those mentioned in the results. Two of the participants passed away after completing their initial follow-up period, due to other medical reasons. One participant had to give up their medical aid due to personal reasons and was not re-evaluated after the initial six weeks.

The participants were responsible for their own transport arrangements as the sonar was done at another centre. At the institution, transport is arranged to and from the various units to the hospital, but no transportation was supplied by the institution to the centre for Duplex doppler evaluation.

Comprehensive follow-up of all participants was achieved thus minimising the possibility of missed outcomes.

\section{Data analysis}

The data was collected prospectively. The surgeon (OK) completed the Modified Caprini Risk Assessment form for every patient. This scoring system is widely tested, and the modified version was used for the 2012 American College of Chest Physicians (ACCP) Guidelines. $^{20}$

The data analysis was done in collaboration with the South African Medical Research Council. The outcome parameter of interest was the presence or absence of DVT. The descriptive statistics method was used to present summary statistics for the mean operative time, the average duration of stay in the hospital and mean time to independent mobilisation. The associated 95\% confidence interval for the summary statistics was also presented. Also, the association between DVT and the demographic and lifestyle characteristics were evaluated using chi-square statistics. STATA 13 was the statistical package of choice.

\section{Results}

Fifty-seven participants (28 males and 29 females) with 30 reverse shoulder replacements, 22 hemiarthroplasties, and five resurfacing shoulder replacements were followed for six weeks (Table I).

The incidence of DVT was $12.3 \%(7 / 57)$ with a $95 \%$ confidence interval $(\mathrm{Cl})[5.1,23.7]$. No fatalities were reported.

Two axillary vein and three brachial vein DVTs accounted for the upper limb DVTs. Two DVTs were reported in the lower leg (four females, three males). All were reported as being of acute onset: two DVTs (one axillary vein; one peroneal vein DVT) were reported to cause partial obstruction of the vein. Incomplete DVTs count as DVTs as they have a similar effect as a complete obstruction on the clotting cascade.

The cardiology department handled the patients' management and dose adjustments for the treatment of a DVT. One patient with axillary vein and one with a brachial vein DVT were symptomatic. The mean age of the participants with a DVT was 63 years with a 95\% Cl [51.8, 74.2] (Table II).
Four participants with a DVT were on thromboprophylaxis at the time of diagnosis. Two of these were on Ecotrin ${ }^{\circledR} 81 \mathrm{mg}$ daily for cardiovascular-related reasons. Two were on Clexane ${ }^{\circledR} 40 \mathrm{mg}$ subcutaneously daily.

Eighteen participants received thromboprophylaxis due to their individual risk factors and not due to their Caprini scores. Of these, nine received adequate dosages of low-molecular-weight heparin $(\mathrm{LMWH})$ at $0.5 \mathrm{mg} / \mathrm{kg}$ according to their body weight. There were no unusual wound complications or bleeding events recorded in the group that received thromboprophylaxis. None of the participants in the study population had a blood transfusion post-operatively.

Each participant reported on their social smoking habits. A total of ten participants smoked, and one of these had a DVT. There was no association demonstrated between smoking and an increased risk of a DVT in the study.

Females with reverse shoulder replacements had a longer post-operative stay (Figure 1).

The average Caprini score for the study population was 5.8 [2; 13]. For the participants with a positive duplex Doppler, the mean was 7.4 [4; 13]; the females with a DVT had an average score of $9.3[7 ; 13]$, and the average score for the males was $5[4 ; 6]$.

Four of the participants in the study had shoulder replacements due to trauma. They were all admitted via the emergency unit and surgery was scheduled for the next available elective list. Three participants received adequate thromboprophylaxis in the form of low-molecular-weight heparin $0.5 \mathrm{mg} / \mathrm{kg}$ daily (treatment was started on the day of admission). Of these four trauma participants, one had a DVT. The participant was on thromboprophylaxis since admission to the hospital and the DVT was symptomatic. The DVT was reported as an acute thrombus of the brachial vein diagnosed on day 3 post-operatively (four days after sustaining the fracture).

The average BMI of the study population was 32.1 [17.9; 51.6] with a mean age of 62.6 [35; 80] years. The participants mobilised independently to a distance of $50 \mathrm{~m}$ on average during the first day after the surgery. The mean operative time was 58.5 minutes $[24 ; 135]$ for all types of replacements with an average length of hospital stay post-operatively of 3.9 days $[1 ; 23]$.

There is no statistically significant difference between the duration of surgery between subjects with a DVT and those without.

One participant who had a DVT of the ipsilateral arm also had an unprovoked DVT of the iliac vein in 2003. This history was obtained at the time of the duplex Doppler studies as the participant forgot about the incident and never had any investigations done to rule out coagulation disorders at the time. This participant had an ipsilateral, symptomatic DVT in the axillary vein diagnosed on the ninth day post surgery. Adequate thromboprophylaxis, in the form of LMWH $0.5 \mathrm{mg} / \mathrm{kg}$, was instituted $48 \mathrm{~h}$ after surgery. The start of the prophylaxis was delayed due to excessive drainage in the Porto-Vac in the first $48 \mathrm{~h}$ post-surgery. The current DVT was reported to be a complete, soft thrombus of $6.8 \mathrm{~cm}$ in length of the axillary vein. The DVT appeared soft and slightly dense; this is in keeping with the appearance of an acute DVT.

\section{Discussion}

Orthopaedic patients are at high risk for both bleeding and DVT and will remain a dilemma for treating surgeons and physicians. ${ }^{21}$ Even though understanding of DVT and PE remains incomplete, a survey of the members of the American Association of Hip and Knee Surgeons reported that $100 \%$ use some form of thomboprophylaxis. ${ }^{22}$ On the contrary, $58 \%$ of the members of the British Elbow and Shoulder Society do not use any routine thomboprophylaxis during shoulder surgery. ${ }^{10}$ However, the more these conditions are investigated, the more additional questions come to the fore. ${ }^{18}$ 


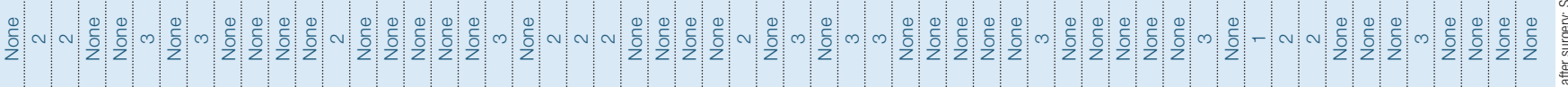

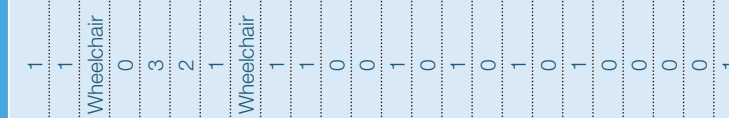

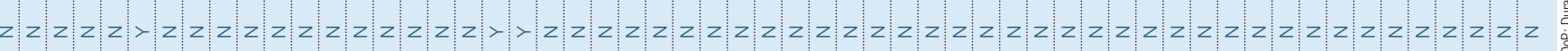

a) manmta

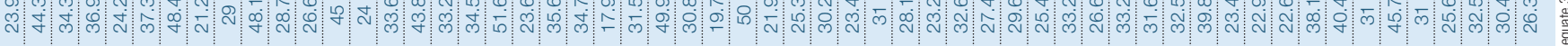

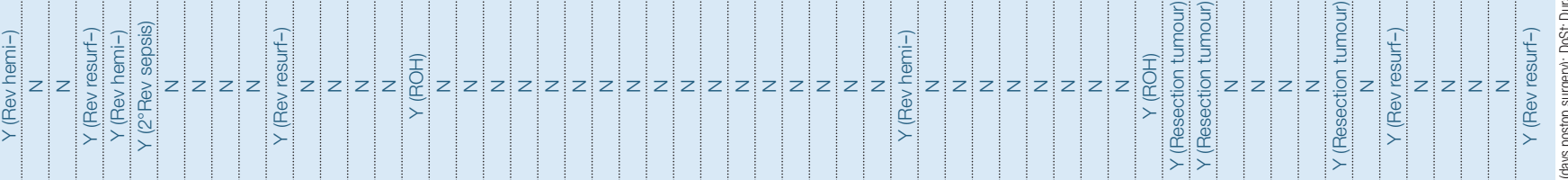


Table II: Subjects with a DVT

\begin{tabular}{|c|c|c|c|c|c|}
\hline Age & Sex & Location & $\begin{array}{l}\text { Time to } \\
\text { diagnosis }\end{array}$ & $\begin{array}{c}\text { Complete/ } \\
\text { partial }\end{array}$ & Prophylaxis \\
\hline 56 & $\mathrm{~F}$ & Axillary & 9 & Complete & LMWH 48 h postop \\
\hline 55 & $\mathrm{~F}$ & Brachial & 9 & Complete & None \\
\hline 43 & M & Axillary & 9 & Partial & Ecotrin (chronic medication) \\
\hline 66 & $\mathrm{~F}$ & Brachial & 3 & Complete & LMWH preop \\
\hline 71 & $\mathrm{~F}$ & Brachial & 9 & Complete & None \\
\hline 74 & M & Tibialis anterior & 10 & Complete & Ecotrin (chronic medication) \\
\hline 76 & M & Peroneal & 10 & Partial & None \\
\hline
\end{tabular}

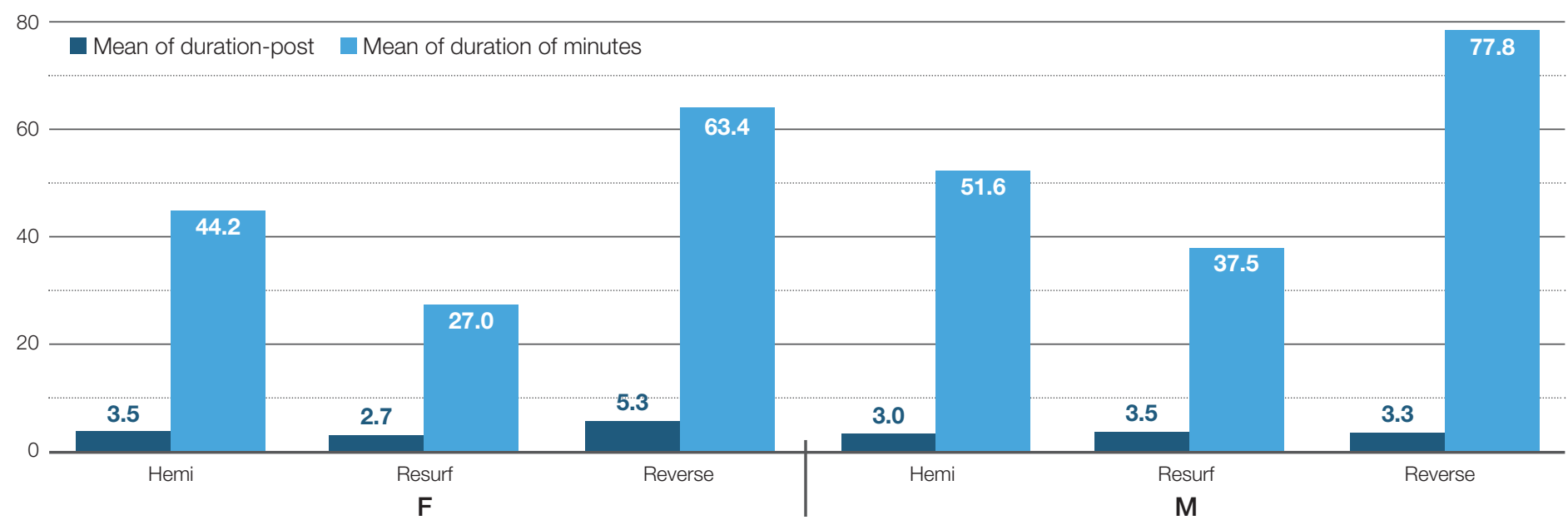

Figure 1. Graph of duration of procedure and stay post-operative by type of replacement and sex

Our data indicates a DVT rate of $12.3 \%$. This value is similar to the $13 \%$ incidence of DVT in Willis and co-workers' series. ${ }^{1}$ The incidence of thromboembolic disease in Farng et al.' ${ }^{5}$ study after shoulder arthroplasty was less than $0.6 \%$ in 15288 shoulders. DVT of the lower extremity after shoulder arthroscopy has rarely been noted in the literature, with reported rates of symptomatic DVT and PE in 65000 patients of less than $0.01 \% .{ }^{23}$

The remaining patient risk factors for sex, age, smoking, and duration of surgery were not statistically significant.

Ultrasound is the modality of choice when upper extremity DVT is suspected. 8,10,18,24 Upper extremity sonographic evaluation of the venous system is more challenging than that of the lower extremity. Attention to detail is critical in areas of duplicated veins to avoid overlooking a thrombus in one of the paired veins. Current literature shows the sensitivity of venous Doppler ultrasound for upper extremity DVT to range from $78 \%$ to $100 \%$ and its specificity ranges from $82 \%$ to $100 \% .{ }^{24-25}$

Ultrasound is a non-invasive investigation and does not result in radiation exposure. On B-mode imaging, acute thrombi may appear in areas of variable echogenicity within the vessel lumen. ${ }^{26}$ The present study made use of the so-called combined modality ultrasound (compression and Doppler ultrasound), as suggested in a recent evidence-based clinical practice guideline. ${ }^{27}$ Compression of a healthy vein completely closes the vessel lumen, whereas the presence of a DVT prevents coaptation of the vein walls. ${ }^{28}$ All of the DVTs reported were acute.

The mean length of surgery was 58.32 minutes for all types of replacements (Figure 1). A review article by Anakwe and co-workers used guidelines from the United Kingdom National Institute for Clinical Excellence (NICE) and interpreted major orthopaedic surgery as upper limb surgery lasting more than 90 minutes. ${ }^{12}$ The recommendation is that this should include the total anaesthetic time. In the current study, the so-called skin-to-skin time was recorded. For shoulder surgery, the time positioned in the beach chair should be taken into account as the patient is supine for the rest of the procedures like the inter scalene block administration.

Multiple guidelines regarding appropriate thromboprophylaxis, in general, have been proposed. There is controversy within the orthopaedic community as to the adequate choice of prophylaxis. ${ }^{3}$ On the other hand, failure to prescribe thromboprophylaxis may be presented as a failure of care. ${ }^{12}$ In-hospital mortality due to VTE is a common avoidable cause of death in trauma patients. ${ }^{29}$ Anticoagulation, on the other hand, is not a benign treatment option. It has significant bleeding complications without reducing clinically relevant symptomatic DVTs, PE and fatal PE rates. ${ }^{30}$ In the current study four participants received some form of prophylaxis and still developed a DVT. Of these medications, most have drawbacks. LMWH and fondaparinux require nonoral administration. The newly introduced direct thrombin inhibitor is an oral agent that does not require monitoring; however, it causes elevation of transaminase levels and an increased rate of coronary events of unclear significance. Factor Xa inhibitor is also taken orally, but there are concerns regarding excessive wound complications. ${ }^{31}$ Vitamin $\mathrm{K}$ antagonists require close monitoring and have unpredictable pharmacokinetics. ${ }^{21}$

The ACCP provides evidence-based recommendations for VTE prevention in some clinical scenarios, but do not advise on prevention of upper extremity DVT. Recommendations broadly focus on the relative risk for thrombosis compared to bleeding. Furthermore, the assessment model provides a selection of mechanical and pharmacological prophylaxis. ${ }^{32}$

The average BMI in our study was 32.1 which is similar to obesity rates in the United States. Adult obesity rates in the US have increased by $129 \%$ in the past decade, with more than $30 \%$ of the 
adult population currently with a BMl of $>30 \mathrm{~kg} / \mathrm{m}^{2}$. The very obese patients pose unique challenges during the procedure regarding optimum positioning in the beach chair, with pendulous bellies limiting the back upright positioning. It also affects the length of surgery. In the post-operative period, they lose an extremity to aid with mobilisation and often mobilise with difficulty. It is not surprising that the patients with a $\mathrm{BMl}$ of $>50 \mathrm{~kg} / \mathrm{m}^{2}$ have a three times higher risk of VTE than the non-obese. ${ }^{33}$

Virchow's triad of hypercoagulability, stasis and intimal damage is the historical foundation for the formation of a thrombus. These factors, in combination with elderly patients in a partially dehydrated state due to overnight starvation, make up the majority of participants in the study population. The physiologic stress response associated with major joint replacement surgery should not be underestimated. Several thrombogenic factors are released intra-operatively, and there is a significant pooling of blood in the lower extremities during the surgery. ${ }^{11}$

The arm is routinely immobilised in a sling after shoulder replacement surgery, which causes stasis that contributes to the occurrence of thrombosis. ${ }^{34}$ There is a relatively lower gravitational stress in the upper extremity compared with lower extremity, and this might explain the lower incidence of DVTs. Prior history of DVT has been consistently demonstrated to be a predominant risk factor for post-operative $\mathrm{DVT}^{6}$ and is the only risk factor recognised in the 2010 guidelines of the American Academy of Orthopaedic Surgeons (AAOS). ${ }^{15,22}$

Complications relating to VTE in shoulder arthroplasty are significantly increased in trauma shoulder replacements. ${ }^{6}$ The risk factors that have been associated with post-operative VTE are age, obesity, trauma, antithrombin III deficiency, protein $S$ and $\mathrm{C}$ deficiency, malignancy, oral contraceptives or hormone replacement therapy, recent myocardial infarction, previous pulmonary embolism or DVT and prolonged immobilisation. ${ }^{35}$

Malignancy is an important risk factor for thrombosis in the upper extremity. ${ }^{36-37}$ Two participants in the current study had a shoulder replacement done for active metastatic cancer. Neither of them had a thromboembolic disease.

Anticoagulation with subcutaneous LMWH (enoxaparin) should be prescribed at a dosage of $0.5 \mathrm{mg} / \mathrm{kg}$. This dose was not complied with, as the standard dosage was Enoxaparin 40 mg daily, even for those participants with a BMl of 50 and weight of $120 \mathrm{~kg}$.

A total of ten participants smoked, of whom one had a DVT. Participants who smoke tend to mobilise sooner and mobilise further to smoking areas. The Caprini Score does not include smoking as a risk factor for a DVT.

Our study has limitations. The sample size is small. Four limb studies were done in $17.5 \%$ of the population. Asymptomatic DVTs of the lower limbs could have been missed. It should be highlighted that complete and comprehensive follow-up was achieved. However, a strength of the study is that all the surgery was performed by two surgeons and all duplex Doppler studies were conducted by the same sonologist. The participants furthermore represent a broad demographic population; this reflects on the scope of practice at the centre.

\section{Conclusion}

The current study demonstrates that the incidence of DVT after shoulder replacement surgery was higher than anticipated and is similar to the DVT rates in lower limb arthroplasty. We failed to predict correctly who would need routine anticoagulation. Even with prophylaxis, some participants developed a DVT. Therefore, we recommend that patients who receive reverse shoulder replacements, trauma replacements, females older than 70 years of age, patients who have tumour resection surgery and those with underlying active metastatic disease should receive routine and adequate thromboprophylaxis.
Due to the increase in BMl in the general population, surgeons should avoid excessive hip flexion when positioning patients in the beach-chair position. This is to prevent the pendulous abdomen from limiting further pelvic venous flow.

Surgeons should individualise those patients not in the above categories when deciding whether to give thromboprophylaxis. The Caprini score is useful and should form part of the consent process.

\section{Acknowledgement}

Dr Koch and Dr Du Plessis would like to acknowledge the South African Orthopaedic Association for a research grant sponsored by Smith \& Nephew. The grant was used in data collection.

\section{Compliance with ethics guidelines}

This study was approved by 1 Military Hospital Research Ethics Committee adhering to Good Clinical Practice/International Conference on Harmonisation and South African Clinical Trial Guidelines. Ethics approval number: IMH/302/6

\section{References}

1. Willis AA, Warren RF, Craig EV, Adler RS, Cordasco FA, Lyman S, et al Deep vein thrombosis after reconstructive shoulder arthroplasty: A prospective observational study. J Shoulder Elbow Surgery 2009;18:100-106. doi:10.16/j.jse.2008.07.011

2. Madhusudhan TR, Sinha A, Widdowson D. Deep vein thrombosis in shoulder arthroplasty- A prospective study. BMC Musculoskelet Disord 2013;14:139. doi:10.1186/1471-2474-14-139

3. Greene JW, Deshmukh AJ, Cushner FD. Thromboembolic complications in arthroscopic surgery. Sports Med Arthrosc Rev 2013;21:69-74. doi:10.1097/jsa.0b013e31828a7e76

4. Rockwood Jr CA, Wirth MA, Blair S. Warning: Pulmonary embolism can occur after elective shoulder surgery- Report of two cases and survey of the members of the American Shoulder and Elbow Surgeons. J Shoulder Elbow Surg 2003;12:628-630. doi:10.1016/s1058-2746(03)00051-x

5. Farng E, Zingmond D, Krenek L, SooHoo NF. Factors predicting complication rates after primary shoulder arthroplasty. J Shoulder Elbow Surg 2011;20:557-63. doi:10.1016/j.jse.2010.11.005

6. Day JS, Ramsey ML, Lau E, Williams GR. Risk of venous thromboembolism after shoulder arthroplasty in the Medicare population. J Shoulder Elbow Surg 2015;24:98-105. doi:10.1016/j.jse.2014.09.025

7. Jiang JJ, Toor AS, Shi LL, Koh JL. Analysis of perioperative complications in patients after total shoulder arthroplasty and reverse total shoulder arthroplasty. J Shoulder Elbow Surg 2014;23:1852-59. doi:10.1016 jj.jse.2014.04.008

8. Kucher NM. Deep-vein thrombosis of the upper extremities. N Engl J Med 2011;364:861-69. doi:10.1056/nejmcp1008740

9. Willis AA, Verma NN, Thornton SJ, Morrissey NJ, Warren RF. Upper extremity deep-vein thrombosis after anterior shoulder dislocation and closed reduction: A case report. J Bone Joint Surg Am 2005;87: 2086-90. doi:10.2016/JBJS.D.02135

10. Dattani R, Smith CD, Patel VR. The venous thromboembolic complications of shoulder and elbow surgery: A systematic review. Bone Joint J 2013;95:70-74. doi:10.1302/0301-620x.95b1.29854

11. Saleem A, Markel DC. Fatal pulmonary embolus after shoulder arthroplasty. J Arthroplasty 2001;16:400-403. doi:10.1054/arth.2001.20546

12. Anakwe RE, Middleton SD, Beresford-Cleary N, McEachan JE, Talwalkar SC. Preventing venous thromboembolism in elective upper limb surgery. J Shoulder Elbow Surg 2013;22:432-38. doi:10.1016/j.jse.2012.10.033

13. Passman MA. Mandated quality measures and economic implications of venous thromboembolism prevention and management. Am J Surg 2010;199:S21-S31. doi:10.1016/j.amjsurg.2009.10.005

14. Eikelboom JW, Karthikeyan G, Fagel N, Hirsh J. American Association of Orthopedic Surgeons and American College of Chest Physicians guidelines for venous thromboembolism prevention in hip and knee arthroplasty differ: What are the implications for clinicians and patients? Chest 2009;135:513-20. doi:10.1378/chest.08-2655

15. Parvizi J, Ghanem E, Joshi A, Sharkey PF, Hozack WJ, Rothman RH. Does 'excessive' anticoagulation predispose to periprosthetic infection? J Arthroplasty 2007;22:S24-S28. doi:10.1016/j.arth.2007.03.007

16. Fender D, Harper WM, Thompson JR, Gregg PJ. Mortality and fatal pulmonary embolism after primary total hip replacement: Results from a regional hip register. J Bone Joint Surg Br 1997;79:896-99. doi: 0301$620 \times / 97 / 67677$ 
17. Watts AC, Howie CR, Simpson AH. Assessment of a self- administration protocol for extended subcutaneous thromboprophylaxis in lower limb arthroplasty. J Bone Joint Surg Br 2006;88:107-10. doi:10.1302/0301620x.88b1.17003

18. Cronan JJ. Deep vein thrombosis: Imaging diagnosis and related controversies. Ultrasound Clin 2011;6:421-33. doi:10.1016/ j.cult.2011.07.002

19. Caprini JA. Risk assessment as a guide for the prevention of the many faces of venous thromboembolism. Am J Surg 2010;199:S3-S10. doi:10.1016/j.amjsurg.2009.10.006

20. Geerts WH, Bergqvist D, Pineo GF, Heit JA, Samama CM, Lassen MR, et al. Prevention of venous thromboembolism: American College of Chest Physicians Evidence-Based Clinical Practice Guidelines (8th Ed). Chest 2008:S381-S453 doi:10.1378/chest.08-0656

21. Tornetta P, Bogdan Y. Pulmonary embolism in orthopaedic patients: Diagnosis and management. J Am Acad Orthop Surg 2012;20:586-95. doi:10.5435/jaaos-20-09-586

22. Lieberman JR, Barnes CL, Lachiewicz PF, Hanssen AD, Clarke HD, Pellegrini VD. Venous thromboembolism debate in joint arthroplasty. J Bone Joint Surg Am 2009;91:S29-S32. doi:10.2106/jbjs.i.00364

23. Jameson SS, James P, Howcroft DW, Serrano-Pedraza I, Rangan A, Reed MR, et al. Venous thromboembolic events are rare after shoulder surgery: Analysis of a national database. J Shoulder Elbow Surg 2011;20:764-70. doi:10.1016/j.jse.2010.11.034

24. Chin EE, Zimmerman PT, Grant EG. Sonographic evaluation of upper extremity deep venous thrombosis. J Ultrasound Med 2005;24:829-38. doi:10.2214/aj.09.2640

25. Weber TM, Lockhart ME, Robbin ML. Upper extremity venous Doppler ultrasound. Radiol Clin N Am 2007;45:513-24. doi:10.1016/ j.rcl.2007.04.005

26. Grant JD, Stevens SM, Woller SC, Lee EW, Kee ST, Liu DM, et al. Diagnosis and management of upper extremity deep-vein thrombosis in adults. Thromb Haemost 2012;108:1097-108. doi:10.1160/th12-050352

27. Bates SM, Jaeschke R, Stevens SM, Goodacre S, Wells PS, Stevenson MD, et al. Diagnosis of DVT: Antithrombotic therapy and prevention of thrombosis, 9th ed: American College of Chest Physicians EvidenceBased Clinical Practice Guidelines. Chest 2012;141:e351S-e418S. doi:10.1378/chest.11-2299

28. Hamper UM, DeJong MR, Scoutt LM. Ultrasound evaluation of the lower extremity veins. Radiol Clin N Am 2007;45:525-47. doi:10.1016/ j.rcl.2007.04.013

29. Stannard JP, Lopez-Ben RR, Volgas DA, Andersonward R., Busbee M, Karr DK, et al. Prophylaxis against deep-vein thrombosis following trauma: A prospective, randomized comparison of mechanical and pharmacologic prophylaxis. J Bone Joint Surg Am 2006;88:261-66. doi:10.2106/jbjs.d.02932

30. Brown GA. Venous thromboembolism prophylaxis after major orthopaedic surgery: A pooled analysis of randomized controlled trials. J Arthroplasty 2009;24:S77-S83. doi:10.1016/j.arth.2009.06.002

31. Jameson SS, Rymaszewska M, James P, Serrano-Pedraza I, Muller SD, Hui AC, et al. Wound complications following rivaroxaban administration: A multicenter comparison with low-molecular-weight heparins for thromboprophylaxis in lower limb arthroplasty. J Bone Joint Surg Am 2012;94:1554-58. doi:10.2106/jbjs.k.00521

32. Kahn SR, Lim W, Dunn AS, Cushman M, Dentali F, Akl EA, et al. Prevention of VTE in nonsurgical patients: Antithrombotic therapy and prevention of thrombosis, 9th ed: American College of Chest Physicians Evidence-Based Clinical Practice Guidelines. Chest 2012;141:e195S226S. doi:10.1378/chest.11-2296

33. Werner BC, Burrus MT, Browne JA, Brockmeier SF. Superobesity (body mass index $>50 \mathrm{~kg} / \mathrm{m}^{2}$ ) and complications after total shoulder arthroplasty: An incremental effect of increasing body mass index. J Shoulder Elbow Surg 2015;24:1868-75. doi:10.1016/j.jse.2015.05.046

34. Flinterman LE, Van DM, Rosendaal FR, Doggen CJM. Current perspective of venous thrombosis in the upper extremity. J Thromb Haemost 2008;6:1262-66. doi:10.1111/j.1538-7836.2008.03017.x

35. Weinmann EE, Salzman EW. Deep-vein thrombosis. N Engl J Med 1994;331:1630-41. doi:10.1056/nejm199412153312407

36. Blom JW, Doggen CJM, Osanto S, Rosendaal FR. Old and new risk factors for upper extremity deep venous thrombosis. J Thromb Haemost 2005;3:2471-78. doi:10.1111/j.1538-7836.2005.01625.x

37. Rathbun SW, Stoner JA, Whitsett TL. Treatment of upper- extremity deep vein thrombosis. J Thromb Haemost 2011;9:1924-1930. doi:10.1111/ j.1538-7836.2011.04466.x 